\title{
The aberrantly expressed miR-372 partly impairs sensitivity to apoptosis in parathyroid tumor cells
}

\author{
Chiara Verdelli1, Irene Forno2,3, Annamaria Morotti2,3, Pasquale Creo4, Vito Guarnieri5, Alfredo Scillitani6, \\ Filomena Cetani7, Leonardo Vicentini8, Gianni Balza9, Edoardo Beretta10, Stefano Ferrero3,11, Valentina Vaira2,3,* \\ and Sabrina Corbetta ${ }^{12, *}$ \\ 1'Laboratory of Experimental Endocrinology, IRCCS Istituto Ortopedico Galeazzi, Milan, Italy \\ 2Department of Pathophysiology and Transplantation, University of Milan, Milan, Italy \\ 3Division of Pathology, Fondazione IRCCS Cà Granda Ospedale Maggiore Policlinico, Milan, Italy \\ ${ }^{4}$ Laboratory of Stem Cells for Tissue Engineering, IRCCS Policlinico San Donato, San Donato Milanese, Milan, Italy \\ 5 Medical Genetics, IRCCS Hospital Casa Sollievo della Sofferenza, San Giovanni Rotondo, Foggia, Italy \\ ${ }^{6}$ Endocrine Unit, IRCCS Hospital Casa Sollievo della Sofferenza, San Giovanni Rotondo, Foggia, Italy \\ ${ }^{7}$ Department of Endocrinology and Metabolism, University of Pisa, Pisa, Italy \\ ${ }^{8}$ Endocrine Surgery, IRCCS Fondazione Cà Granda Ospedale Maggiore Policlinico, Milan, Italy \\ ${ }^{9}$ Endocrinology Unit, Ospedale Manzoni, Lecco, Italy \\ ${ }^{10}$ Endocrine Surgery, IRCCS Ospedale San Raffaele, Milan, Italy \\ ${ }^{11}$ Department of Biomedical, Surgical and Dental Sciences, University of Milan, Milan, Italy \\ ${ }^{12}$ Endocrinology Unit, Department of Biomedical Sciences for Health, University of Milan, IRCCS Istituto Ortopedico Galeazzi, Milan, Italy
}

Correspondence should be addressed to V Vaira or S Corbetta: valentina.vaira@unimi.it or sabrina.corbetta@unimi.it

*(V Vaira and S Corbetta contributed equally to this work)

\begin{abstract}
Parathyroid tumors deregulate microRNAs belonging to the two clusters on the chromosome 19, the C19MC and miR-371-373 clusters. Here, we report that the embryonic miR-372 is aberrantly expressed in half of parathyroid adenomas (PAds) in most of atypical adenomas and carcinomas $(n=15)$. Through in situ hybridization, we identified that miR-372-positive parathyroid tumor cells were scattered throughout the tumor parenchyma. In PAd-derived cells, ectopic miR-372 inhibited the expression of its targets CDKN1A/p21 and LATS2 at both mRNA and protein levels. Although the viability of parathyroid cells was not affected by miR-372 overexpression, the miRNA blunted camptothecin-induced apoptosis in primary PAd-derived cultures. miR-372 overexpression in parathyroid tumor cells increased parathormone (PTH) mRNA levels, and it positively correlated in vivo with circulating PTH levels. Conversely, the parathyroid-specific genes TBX1 and GCM2 were not affected by miR-372 mimic transfection. Finally, miR-372 dampened the Wnt pathway in parathyroid tumor cells through DKK1 upregulation. In conclusion, miR-372 is a novel mechanism exploited by a subset of parathyroid tumor cells to partially decrease sensitivity to apoptosis, to increase PTH synthesis and to deregulate Wnt signaling.
\end{abstract}

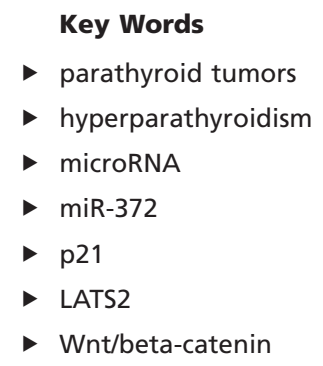

Endocrine-Related Cancer (2018) 25, 761-771 


\section{Introduction}

Parathyroid tumors are almost invariably associated with parathormone (PTH) hypersecretion, which defines the endocrine disease hyperparathyroidism. Primary hyperparathyroidism is associated with bone and kidney complications. Though mainly benign, parathyroid tumor-related hyperparathyroidism occurs in up to $2 \%$ of post-menopausal women, making it the third most common endocrine disorder following diabetes and thyroid diseases. Parathyroid tumorigenesis is far from being elucidated and alternative treatments to surgery are limited. Similar to what was observed in most human cancers, microRNAs have been shown to be involved in parathyroid tumorigenesis (Corbetta et al. 2010, Vaira et al. 2016). Indeed, we previously described deregulated expression of chromosome 19 microRNA clusters, the C19MC and miR-371-373, in parathyroid tumors from patients with primary hyperparathyroidism. Opposite to parathyroid carcinomas (PCas), amplification of C19MC and of distal miR-371-373 locus was observed only in a minority of parathyroid adenomas (PAds). (Vaira et al. 2012a). Given the importance of the miRNA cluster miR-371-373 in embryogenesis, development and tumorigenesis (Langroudi et al. 2015), we sought to better characterize the role of one of its member, the miR-372, in parathyroid tumors.

miR-372 is one of the most abundant microRNAs identified in human blastocysts (Rosenbluth et al. 2013) and the human homologue of the embryonicspecific murine miR-290-295 cluster (Medeiros et al. 2011). The expression of this family of microRNAs decreases as embryonic stem cells (ESCs) differentiate (Medeiros et al. 2011). Furthermore, miR-372 enhances reprogramming of human somatic cells by increasing the kinetics of mesenchymal-to-epithelial transition during reprogramming and blocks transforming growth factor $\beta$-induced epithelial-to-mesenchymal transition of human epithelial cells (Subramanyam et al. 2011). The miR-371-373 cluster expression is also restricted to human ESCs in physiological conditions (Suh et al. 2004). Indeed, microRNAs of the cluster miR-371-373 are frequently deregulated in several human neoplasias in which they influence the replicative potential (Di Leva et al. 2014).

In this study, we aimed to get insights into miR-372 deregulation in different histotypes of parathyroid tumors, and understanding which targets and signaling are mostly affected by miR-372 modulation in primary parathyroid tumor cultures.

\section{Materials and methods}

\section{Parathyroid tissues}

Formalin-fixed paraffin-embedded (FFPE) samples were collected from 15 PCas, 19 parathyroid atypical adenomas (PAts), 46 PAds and six normal parathyroid glands incidentally removed from normocalcemic patients treated with thyroid surgery. Specimens from PCas were sampled from the central portion of the tumors to avoid the contamination of parathyroid tissue with adjacent nonparathyroid tissue. PCas samples were from the previously described series (Vaira et al. 2012a). To avoid thyroid tissue contamination, normal parathyroid specimens were microdissected from FFPE sections of thyroid resections using a LMD6000 instrument (Leica Microsystems, Milan, Italy). Histologic classification of PCas was performed according the World Health Organization-published guidelines (Bondenson et al. 2004). In all patients, fasting serum total calcium, phosphate and creatinine were measured by a multichannel autoanalyzer. Intact PTH was determined by a chemiluminescent immunoassay (Nichols Advantage, Nichols Institute Diagnostics, San Clemente, CA, USA) to diagnose primary hyperparathyroidism. Patients' clinical and biochemical features are reported in Supplementary Table 1 (see section on supplementary data given at the end of this article). All carcinomatous and adenomatous specimens were from patients with primary hyperparathyroidism. Fresh samples from additional 25 PAds were collected immediately after surgery, partly snap frozen and partly dissociated for cell culture. This study was approved by an Institutional Ethical Committee (Ospedale San Raffaele Ethical Committee, oSR EC), and patients' informed consent was obtained from all patients.

\section{RNA purification}

Total RNA from FFPE samples was purified using the AllPrep DNA/RNA FFPE Kit (Qiagen), whereas TRIzol reagent (Invitrogen, Thermo Fisher Scientific) was used to isolate RNA from fresh/frozen samples. DNA contamination was removed by DNase I (Life Technologies, Thermo Fisher Scientific) treatment and DNA-free RNA was quantified by spectrophotometry at $260 \mathrm{~nm}$.

\section{MicroRNA detection}

Analysis of miR-372-3p expression, hereafter named miR-372, was carried out on total RNA extracted from 
normal and tumor parathyroid FFPE tissue sections, HEK293 or MCF-7 cultures and PAd-derived cells. The reverse transcription was performed using the microRNA Reverse Transcription Kit with gene-specific primers following manufacturer's specifications. Quantitative Real-Time RT-PCR (qPCR) was conducted on a StepOne Plus System using the TaqMan microRNA assays RNU48-ID001006 and miR-372-3p-ID000560. miR-372 expression level was normalized to the housekeeping RNU48 transcript. Amplification data were collected and analyzed with StepOne Plus v2.3 Software. All reagents and instruments were from Thermo Fisher Scientific.

\section{Gene expression analysis}

Total DNA-free RNA (300 ng) was reverse transcribed using the iScript cDNA Synthesis Kit (Bio-Rad). Then, cDNA was amplified using TaqMan gene expression assay and a StepOne Plus System. The following assays were used: CDKN1A Hs00355782_m1, LATS2 Hs00324396_m1, PTH Hs00757710_g1, TBX1 Hs00271949_m1, GCM2 Hs00899403_m1, AXIN2 Hs00610344_m1, DKK1 Hs00183740_m1， CCND1 Hs00765553_m1. The reference genes HMBS and B2M (Hs00609297_m1 and Hs99999907_m1, respectively) were used to normalize expression data and to obtain relative quantities using the $2^{-\mathrm{DCt}}$ formula. All reagents and instruments were from Thermo Fisher Scientific.

\section{Protein extraction and western blot analysis}

Cells were homogenized using a Nuclear Extract kit (Active Motif, Carlsbad, CA, USA) supplemented with protease and phosphatase inhibitors to obtain both cytoplasm and nuclear protein fractions. Forty micrograms of nuclear or cytoplasmic lysate per sample were then separated by SDS-PAGE, transferred to nitrocellulose membrane (Bio-Rad) and probed with the following primary antibodies: LATS2 (\#13646), p21 Waf1/Cip1 (12D1) (\#2947) (all by Cell Signaling). Histone H3 (ab1791, Abcam) and vinculin (clone V-11-5, Sigma, Merk KGaA) were used as controls for nuclear to cytoplasmic fractions separation and protein loading. Binding of appropriate HRP-conjugated secondary antibodies was revealed using the chemiluminescence ChemiDoc XRS System (Thermo Fisher Scientific). Analyses of bands densitometry were performed using ImageJ software, and protein expression levels were normalized using vinculin as reference.

\section{miRNA in situ hybridization}

In situ hybridization (ISH) analyses were carried out on human parathyroid FFPE tissues using mercury LNA microRNA ISH Optimization kit following the manufacturer's protocol. Specifically, a 5'- and 3'-digoxigenin (DIG)-labeled miR-372-3p (catalog number 38177-15; probe concentration $80 \mathrm{nM}$ ), miR-126-3p (from microRNA ISH Optimization kit five for FFPE), scrambled miRNA control and a 5'-DIG-labeled U6 miRCURY LNA probes (all from Exiqon, Denmark) were used. Staining for miR-126-3p or U6 was used as a positive control, whereas the scrambled probe was used as a negative control. Sections of colon cancer, a tissue known to overexpress miR-372, were also used as positive control. Hybridization, washing and detection procedures were carried out according to the manufacturer's instructions, and reaction was visualized by light microscopy.

\section{Primary parathyroid tumors-derived and commercial cell cultures}

Samples from PAds were cut into $1 \mathrm{~mm}^{3}$ fragments, washed with PBS and partially digested with $2 \mathrm{mg} / \mathrm{mL}$ collagenase type I (Worthington, Lakewood, NJ, USA) for $90 \mathrm{~min}$. Digested tissues were filtered with a cell strainer ( $100 \mu \mathrm{m}$ Nylon, BD Falcon, Rignano Flaminio, Italy) to obtain a single cell suspension and cultured in DMEM supplemented with $10 \%$ fetal bovine serum, $2 \mathrm{mmol} / \mathrm{L}$ glutamine and $100 \mathrm{U} / \mathrm{mL}$ penicillin-streptomycin.

The human breast cancer MCF-7 and human embryonic kidney HEK293 cell lines (ATCC \#HTB22 and CRL1573) were used as controls in miRNA modulation experiments. These cells express very low basal levels of miR-372. All cells were maintained at $37^{\circ} \mathrm{C}, 5 \% \mathrm{CO}_{2} /$ air in a humidified incubator.

\section{Cells transfection}

For miRNA mimic transfection experiments, MCF-7 and HEK293 cells were cultured until they reached $50 \%$ confluence. Conversely, PAd-derived cells were let adhere to cell plates for $48 \mathrm{~h}$ and then transfected with a miR-372 mimic (hsa-miR-372-3p, cat. n. 38177-15) or a tetrachlorofluorescein (FAM)-labeled negative control mimic (AM17121) with Lipofectamine 2000 (all from Thermo Fisher Scientific) following the manufacturer's instructions. Six hours after transfection, the mixes were removed and fresh medium was added. Forty-eight hours later, cells were collected for functional experiments. 
For LATS2 knock-down, HEK293 cells were seeded as before and transiently transfected using Lipofectamine 3000 in OptiMEM media (both from Thermo Fisher Scientific) with either an esiRNA targeting LATS2 (EHU055781, Sigma-Aldrich) or the Universal Negative Control (SIC001, Sigma-Aldrich). LATS2 silencing was evaluated by western blot after $48 \mathrm{~h}$ to confirm antibody specificity (Supplementary Fig. 1).

\section{Luciferase reporter assay}

HEK293 cells were co-transfected in a six-well plate with $1 \mu \mathrm{g}$ of control, p21 or LATS2 3'UTR luciferase reporter plasmid (all from GeneCopoeia Inc., Rockville, MD, USA) and either $100 \mathrm{ng}$ of non-targeting miRNA (control mimic) or miR-372 mimic as indicated. Forty-eight hours post transfection, cells were lysed in LBL lysis buffer, and Firefly/Renilla luciferase reporters' activity was detected using a Dual-Luciferase Reporter Assay System (Promega Corp.) and measured using a luminometer (GloMax, Promega) as described (Vaira et al. 2012b). Firefly to Renilla luciferases relative levels are expressed relative to a control sample (set equal to 1 ). All experiments were repeated at least three times.

\section{Apoptosis analysis}

Apoptotic cell death was measured using the FITC-labeled Annexin V (Annexin V-FITC) apoptosis detection kit (BD Biosciences, Vienna, Austria). Briefly, PAd-derived, MCF-7 orHEK293 transfected cells were incubated with $4.8 \mu \mathrm{M}$ camptothecin (CPT; Sigma-Aldrich) or vehicle for $4 \mathrm{~h}$. Then, cells were collected, washed twice with cold PBS, resuspended in $1 \times$ binding buffer and stained with Annexin V-FITC/PI in the darkness. The percentage of apoptotic cells was quantified by flow cytometry (Navios Flow Cytometer; Beckman Coulter, Fullerton, CA, USA).

\section{Statistical analysis}

miR-372 expression in FFPE sections was presented as relative quantities data \pm S.D. (fold changes over mean of normal parathyroid samples). Normally distributed data were presented as mean \pm s.E.M., whereas not normally distributed values (failure of Kolmogorov-Smirnov test), were $\log 2$ transformed. Differences or correlation between two variables were tested with Mann-Whitney $U$ or Pearson correlation coefficient tests, as appropriate, whereas three or more sample groups were analyzed using one-way ANOVA followed by Bonferroni's correction post-test. A probability value $(P)$ less than 0.05 was considered statistically significant. Statistical analyses were performed using Prism v6.0 (GraphPad Inc).

\section{Results}

miR-372 expression in human parathyroid tissues

From a previous work (Vaira et al. 2012a), we knew that miR-371 and miR-373 were almost undetectable in all parathyroid tissues. Therefore, we focused our efforts on miR-372. Confirming and extending previous data, we found that miR-372 was overexpressed in most parathyroid tumors compared to normal glands, and specifically in 23 out of 46 (50\%) PAds, 14 out of 19 (74\%) atypical PAds and in 11 out of 15 (73\%) PCas (Fig. 1A). More interestingly, miR-372 expression in parathyroid tumors was also higher than in at term placenta, which represents the only human adult tissue physiologically expressing the miRNA. Comparing with the mean expression level detected in at term placentas $(n=3)$, similar miR-372 expression levels were limited to one atypical PAd and 1 PCa (Fig. 1B). Finally, in atypical PAds and PCas, miR-372 levels were significantly higher than in the more indolent PAds ( $P=0.003$ and $P=0.04$, respectively; Fig. $1 \mathrm{~B}$ ).

\section{miR-372 is expressed in PAd epithelial cells}

ISH with controls or a specific probe against miR-372 was performed on PAd FFPE sections to identify cells expressing miR-372. The nuclear U6 and the endothelialspecific miR-126 were used as internal controls in PAd tissues (Fig. 1C, panel a and b). Then, miR-372 localization was investigated in the same parathyroid tumors and in a colorectal cancer tissue. The latter was used as positive control for miR-372 presence (Strauss et al. 2006). As expected, the colorectal cancer displayed diffuse miR-372 staining within the tumor glands (Fig. 1C, panel c). In PAds, scattered miR-372-positive tumor cells within the tumor parenchyma could be identified. In these cells, miR-372 seemed predominantly expressed in the nucleus (Fig. 1C, panel e, arrows). The staining was specific since the scrambled probe did not provide any signal in both colorectal and parathyroid tumors (Fig. 1C, panel d and f).

\section{miR-372 targets p21/CDKN1A and LATS2 genes in parathyroid tumor cells}

The cell cycle regulators cyclin-dependent kinase inhibitor 1A (p21/CDKN1A) and large tumor suppressor 

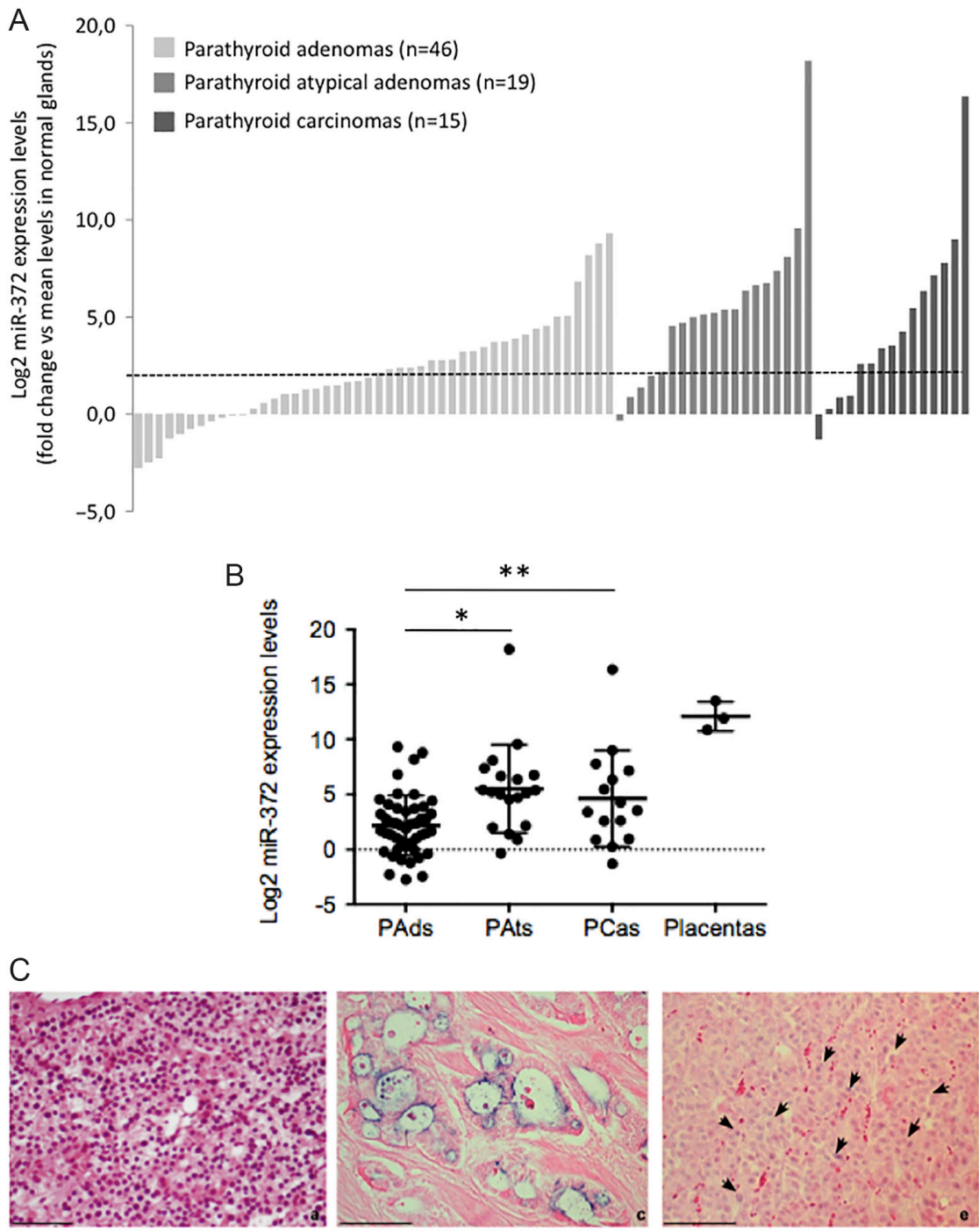

\section{Figure 1}

miR-372 expression levels in parathyroid tissues. (A) miR-372 expression was assessed by qPCR in individual PAds, atypical PAds (PAts) and in PCas tissues; dashed line indicates the threshold set for miR-372 overexpression. (B) Comparison of miR-372 expression levels among parathyroid tumor histotypes (PAds, PAts and PCas) and human at term placentas $(n=3)$. Median with IQR are shown for every group (PAds: 2.0 (IQR 0.2-3.7); PAts: 5.2 (IQR 2.1-6.7); PCas: 3.5 (IQR $0.9-7.1)) ; * P=0.003 ; * * P=0.04$. (C) Representative images of in situ hybridization with positive controls (a, nuclear snRNAU6 and b, endothelialspecific miR-126-3p), a miR-372-3p directed (c, e) or scramble $(d, f)$ probe in FFPE sections of a colon (c, d) or a parathyroid adenoma (a, b, e, f) tissues are shown. Scale bar, $100 \mu \mathrm{m}$. Arrow heads in panel e indicate miR-372-positive parathyroid cells. FFPE, formalin-fixed paraffin-embedded; IQR, interquartile range; PAd, parathyroid adenoma; PAt, parathyroid atypical adenoma; $\mathrm{PCa}$, parathyroid carcinoma; $\mathrm{qPCR}$, quantitative PCR.

kinase 2 (LATS2) are known targets of miR-372 in human ESCs (Qi et al. 2009) and in testicular germ cell tumors (Voorhoeve et al. 2006). Moreover, they have been further confirmed as miR-372 targets in nasopharyngeal (Tan et al. 2014), ovarian (Guan et al. 2017) and colorectal (Yamashita et al. 2012) cancer cells. Based on these considerations, we tested the hypothesis that CDKN1A and LATS 2 could be regulated by miR-372 in parathyroid tumor cells.

Firstly, we assessed whether p21 and LATS2 were direct targets of miR-372. Co-transfection of p21 or LATS2-3'UTR with miR-372 mimic significantly repressed the reporter activity compared to controls $(P=0.001)$ (Fig. 2A).

Transfection of PAd-derived cells $(n=8)$ with a miR-372 mimic (Supplementary Fig. 2A) induced a significant reduction of CDKN1A mRNA levels (Fig. 2B). At variance with HEK293 and MCF-7 cells (Supplementary Fig. 2B and C), miR-372 overexpression in PAd-derived cells significantly reduced LATS2 transcript also (Fig. 2B). The inhibitory effect of miR-372 on p21 and LATS2 was confirmed in PAd-derived and HEK293 cells at protein level (Fig. 2C and Supplementary Fig. 2D). 
A

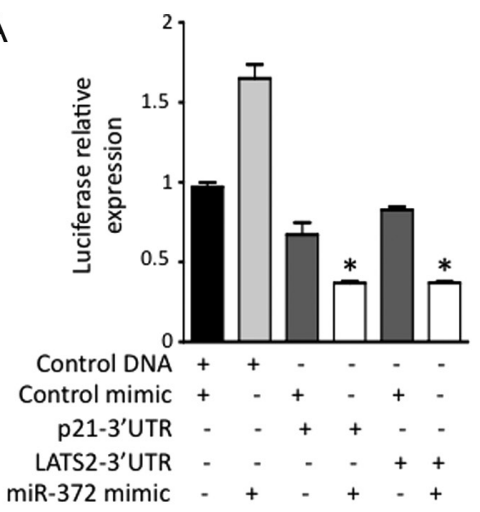

B
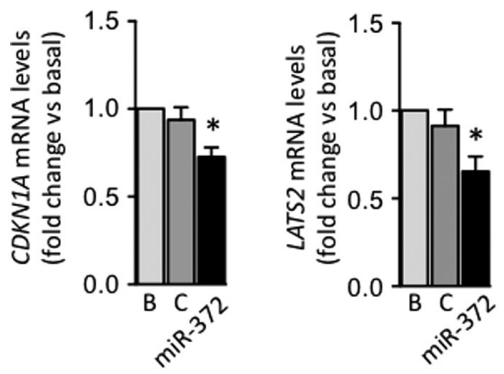

miR-372 protects PAd cells from therapeutic-induced apoptosis

Given that miR-372 regulates the cell cycle genes p21 and LATS2, we investigated the effect of miR-372 overexpression on cell cycle progression of PAd-derived cells. Results showed that cell cycle phases were unaffected by miR-372 modulation in these slowly proliferating primary parathyroid cultures $(1.5 \%$ of cells in $\mathrm{S}$ phase; Fig. 3A and B). Conversely, miR-372 overexpression in the more proliferating HEK293 and MCF-7 cultures (15\% and $4 \%$ of cells in $\mathrm{S}$ phase, respectively) increased the proportion of cells in the $\mathrm{G} 2 / \mathrm{M}$ phase (Supplementary Fig. 3A, B and C).

Next, we investigated whether miR-372 could have a role in regulating cell death rather than viability in PAdderived cultures. Therefore, primary parathyroid tumor cultures transfected with the miRNA or with a mock mimic were treated with the chemotherapeutic CPT or with the vehicle. A modest, yet significant $(P=0.003$; Fig. 3C and $\mathrm{D}$ ), increase in annexin V-positive (apoptotic) cells upon CPT treatment could be observed in PAd cultures. Interestingly, miR-372 overexpression prevents this increase and restored basal levels of cell death $(P=0.02$; Fig. $3 \mathrm{C}$ and $\mathrm{D})$. In line with this result, CDKN1A transcript expression increased after CPT treatment and was partially blunted by miR-372 presence $(P=0.05$; Fig. $3 E)$. Finally, miR-372 also protected HEK293 cells from chemotherapyinduced cell death ( $P=0.01$; Supplementary Fig. 3D).

miR-372 modulates parathyroid-specific genes and Wnt pathway member expression

To get insights into miR-372 potential role in parathyroid pathology, we investigated whether the expression of the transcription factors and master regulators of parathyroid development TBX1 (Verdelli et al. 2017) and GCM2 (Guan et al. 2016), of the parathormone gene PTH, or of the Wnt pathway regulators Axin2, DKK1 and CCND1 was affected by the ectopic overexpression of the miRNA in primary parathyroid cultures.

Both TBX1 and GCM2 expression levels were unaffected by the presence of miR-372 (Fig. 4A and B). Conversely, PTH levels were significantly upmodulated in the presence of miR-372 overexpression (Fig. 4C). In line with this finding, miR-372 levels in PAd tissues were positively correlated with circulating parathyroid hormone levels $\left(r^{2}=0.119, P=0.003\right.$; Fig. 4D) of the patients.

Finally, we tested the hypothesis that miR-372 modulated the expression of genes of the Wnt pathway,
(C) 2018 Society for Endocrinology Published by Bioscientifica Ltd. Printed in Great Britain 
A

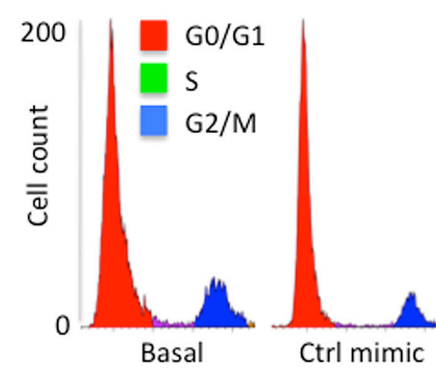

C

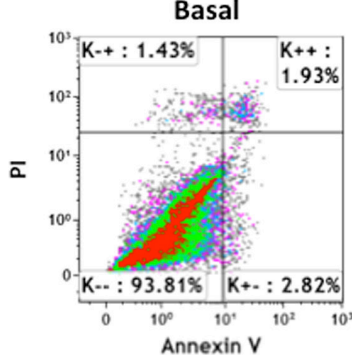

Basal + CPT $4.8 \mu \mathrm{M}$

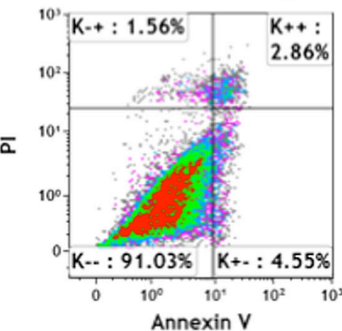

D

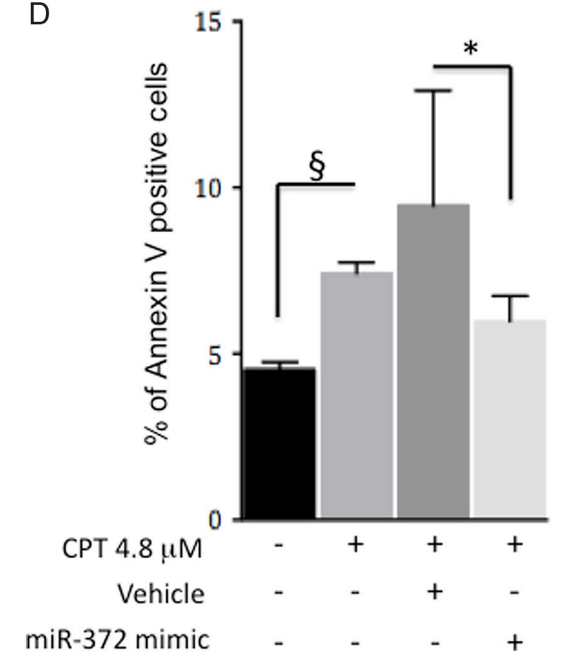

B
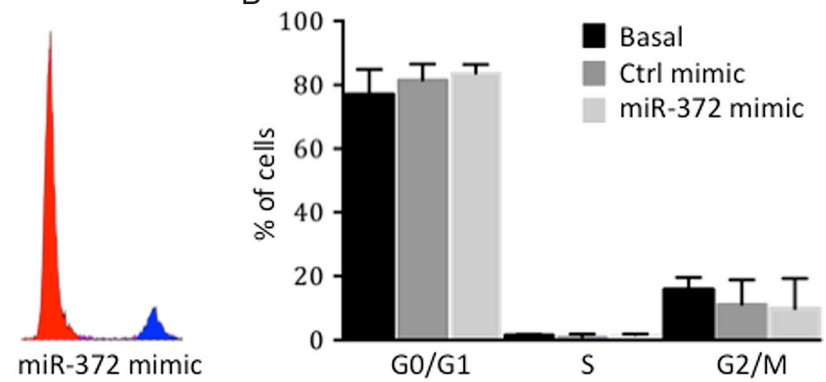

Vehicle

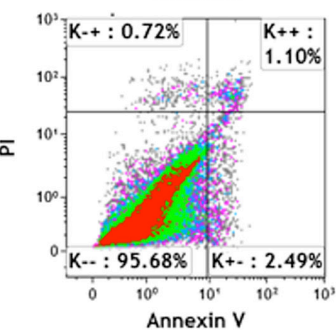

Vehicle + CPT $4.8 \mu \mathrm{M}$

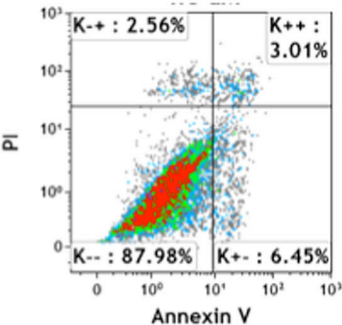

miR372 mimic $60 \mathrm{nM}+$ CPT $4.8 \mu \mathrm{M}$

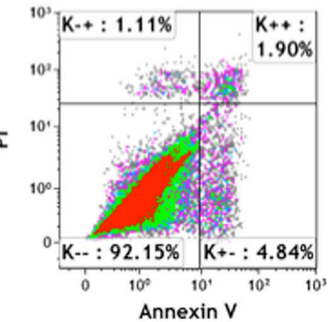

E

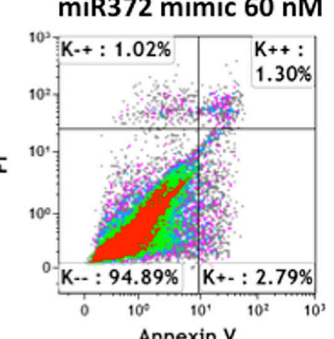

$+1$ $\bar{a}$
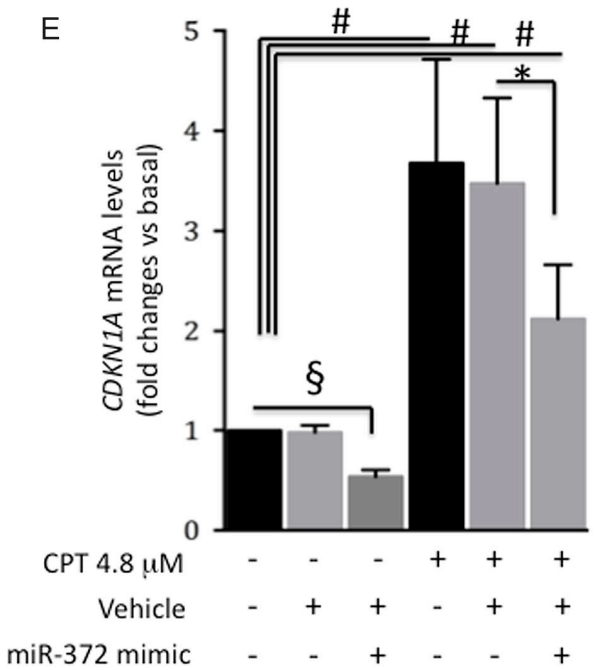

Figure 3

Effect of miR-372 overexpression on PAd-derived cells viability. (A) Cell cycle progression was assessed in PAd-derived cells transfected with a miR-372, a control mimic or left untreated (basal) by propidium iodide (PI) staining and FACS analysis. A representative cell cycle analysis is shown.

(B) Quantification of cell cycle phases (obtained as in A) from three primary PAds-derived cell cultures. Bars, mean \pm s.E.M. (C) Apoptotic cell death was analyzed using Annexin V/PI staining and FACS analysis in primary PAd cultures transfected with the indicated miRNA mimic after camptothecin $(\mathrm{CPT} ; 4.8 \mu \mathrm{M})$ or vehicle treatment $(4 \mathrm{~h})$. A representative experiment is shown and the percentages of PI-positive, Annexin $\mathrm{V}$-positive or double-positive cells are indicated in each graph. (D) Quantification of annexin V- and annexin V/PI-positive PAd cells from three independent experiments treated as described in panel C. Bars, mean \pm s.E.M.; ${ }^{\S} P=0.003 ;{ }^{*} P=0.02$ by Mann-Whitney $U$ test. (E) CDKN1A/p21 mRNA levels were evaluated by qPCR in PAd samples treated as in $C$. The target level in untreated controls was set equal to 1 and data in samples are expressed as fold-differences. Bars, mean with S.E.M. $(n=3) .{ }^{\S} P=0.038 ; \# P=0.04 ;{ }^{*} P=0.05$ by ANOVA. PAd, parathyroid adenoma; qPCR, quantitative PCR. 
as previously reported (Zhou et al. 2012). Opposite of what we observed in HEK293 cells (Fig. 4E), miR-372 overexpression in PAd-derived cells did not modulate AXIN2 expression (Fig. 4F). More interestingly, in PAd cultures, miR-372 mimic transfection increased DKK1 and decreased CCND1 transcripts (Fig. 4F). This signaling was specific for miR372 since miR-371 levels were unaffected (Fig. 4G). Overall, these data were consistent with the limited proliferative nature of PAds and suggested that the Wnt signaling may be inhibited in the subset of miR-372expressing parathyroid tumor cells.

\section{Discussion}

Our data highlight that the embryonic-specific miR-372 (Li et al. 2009), belonging to the miR-371-373 cluster, is deregulated in parathyroid tumors. Importantly, this miRNA is progressively overexpressed in the more aggressive atypical PAds and in carcinomas compared to PAds. This is in line with previous finding of C19MC
miRNA deregulation in parathyroid diseases (Vaira et al. 2012a) and underscores that embryonic or developmental pathways are reactivated and exploited by parathyroid tumor cells. Furthermore, highlighting the potential role of miR-372 in parathyroid tumorigenesis, miR-372 expression in tumor tissues correlates with increased circulating levels of parathyroid hormone in patients with PAds.

In parathyroid tumor cells, miR-372 represses the cell cycle genes CDKN1A/p21, LATS2 and cyclin D1 thereby explaining the slow proliferating nature of this neoplasm, and potentially protects parathyroid cells from apoptotic stimuli, such as exposure to chemotherapeutics.

miR-372 has been shown to play a substantial role in several human cancers, where it is either upregulated (Voorhoeve et al. 2006, Cho et al. 2009, Yamashita et al. 2012, Li et al. 2013, Chen et al. 2015, Tu et al. 2015, Liu et al. 2016) or downregulated (Tian et al. 2011, Huang et al. 2015, Wu et al. 2015, Liu et al. 2016). Our data show that miR-372 is aberrantly expressed in a half of
A

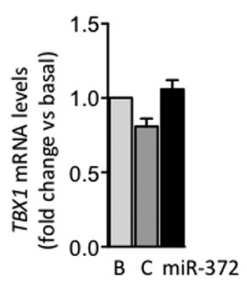

B
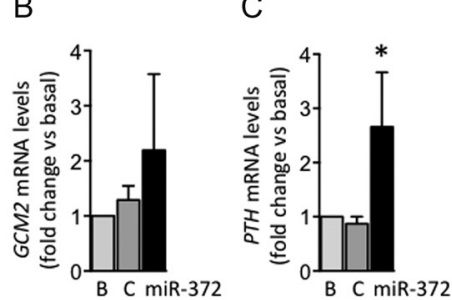

D

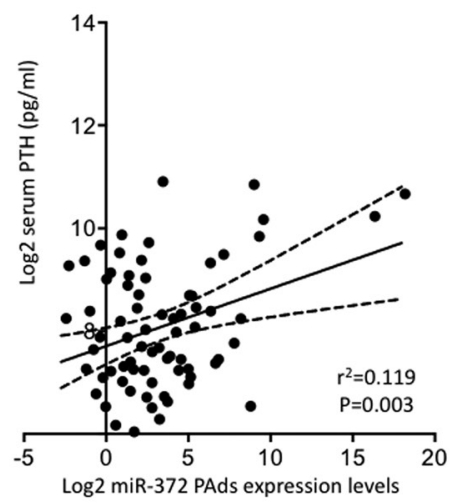

E HEK293 cells with miR-372 mimic for 48 hours
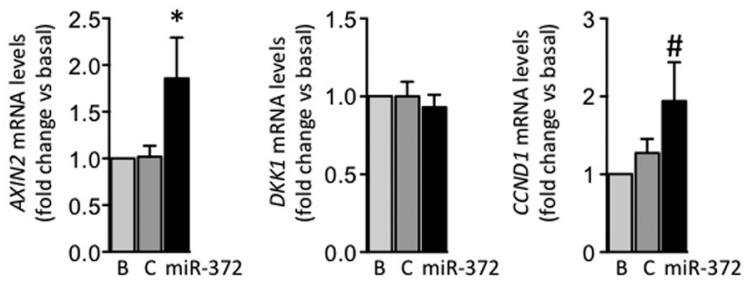

$\mathrm{F}$
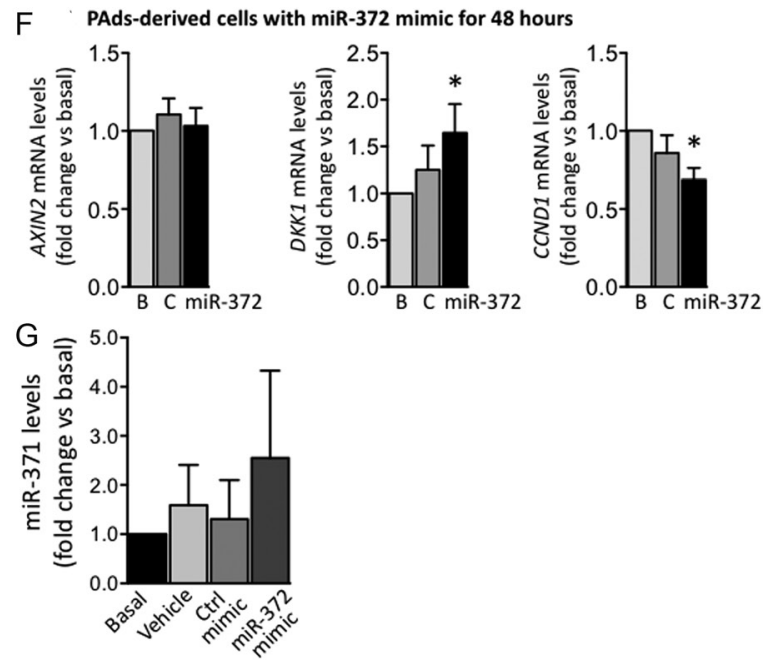

Figure 4

miR-372 modulation of parathyroid-specific and Wnt-related genes signaling in PAd-derived cells. (A, B and C) The parathyroid-specific TBX1, GCM2 and PTH genes levels were investigated in PAd-derived cultures transfected with a miR-372 or a control (C sample) mimic or left untreated (B sample) by qPCR. $* P=0.04$. (D) Circulating PTH levels were correlated with tumor miR-372 expression in patients with parathyroid tumors $(n=73)$. A $y=6$ value corresponds to the log2 upper limit of the normal range of circulating PTH $(65 \mathrm{pg} / \mathrm{mL})$. Samples correlation was assessed using the Spearman correlation coefficient analysis. (E and F) The Wnt-related AXIN2, DKK1 and CCND1 genes levels were investigated in HEK293 cells (E) or PAd primary cultures (F) transfected as in A-C by qPCR. ${ }^{*} P<0.05, \# P=0.01$; $\mathrm{B}$, basal; $\mathrm{C}$, control mimic; miR-372, miR-372 mimic. (G) miR-371 expression was analyzed by qPCR in the indicated PAd-derived cells $(n=3)$. Bars, mean \pm s.E.M. PAd, parathyroid adenoma; PTH, parathormone; qPCR, quantitative PCR. 
PAds and in the majority of both atypical PAds and PCas, where it is also present at significantly higher levels than in PAds. Therefore, aggressive behavior in parathyroid tumors appears to be associated with enriched expression of the embryonic miR-372, similar to what was reported for glioma, colorectal and oral cancers (Yamashita et al. 2012, Chen et al. 2015, Tu et al. 2015). In this context, it is noteworthy that PAts showed a miR-372 expression pattern close to that detected in PCas.

From a histological point of view, only a subset of parathyroid epithelial tumor cells scattered throughout the tumor parenchyma expressed miR-372. Neither cell clusters, nor association with vessels was observed, while stromal endothelial cells and fibroblasts did not express miR-372. Therefore, we can presume that there is a certain degree of intra-tumor heterogeneity at least for miR-372 expression in parathyroid tumors, as also described for other neoplastic contexts (Eriksen et al. 2016).

To get insights of miR-372 signaling in parathyroid tumorigenesis, we derived short-term primary tumor cultures from PAd tissues. This model has important limitations, such as the slow proliferating nature of the cultures and the limited amount of viable cells available after tissue dissociation. Nevertheless, it represents the only cellular model of parathyroid tumorigenesis available. Using this in vitro model, we showed that miR-372 represses CDKN1A/p21 and LATS2, two genes of particular interest for parathyroid tumorigenesis. Indeed, $C D K N 1 A / \mathrm{p} 21$ is a multifaceted molecule, playing multiple critical roles in cell cycle regulation, differentiation, apoptosis, DNA repair, senescence, aging and stem cell reprogramming (Cho et al. 2009). CDKN1A/p21 is a member of the cyclindependent kinase inhibitors, which have been found to be variably downregulated in parathyroid tumors. Despite data about $C D K N 1 A / \mathrm{p} 21$ in parathyroid tumors are controversial (Arribas et al. 2000, Stojadinovic et al. 2003, Buchwald et al. 2004, McCoy et al. 2015), recent evidence suggests that p21 is reduced in parathyroid tumors. Considering the role of p21 in cell death regulation (Gartel et al. 2005), miR-372 may contribute to parathyroid tumorigenesis inhibiting apoptosis through p21 repression. Indeed, miR-372 overexpression in PAdsderived cells partially reduced the percentage of apoptotic cells in presence of the chemotherapeutic agent CPT.

LATS2 is a component of the Hippo signaling pathway, which has never been explored in parathyroid tumors. In mammals, the Hippo pathway functions through a kinase cascade that ultimately regulates the expression of genes involved in cell proliferation and survival signaling (Zhao et al. 2008). Furthermore, decreased expression of LATS2 lowers the ability of p53 to induce p21 and cell cycle arrest (Zhao et al. 2008).

Finally, miR-372 has been reported to modulate the Wnt/ $\beta$-catenin pathway (Zhou et al. 2012). The Wnt/ $\beta$-catenin components are epigenetically and genetically deregulated in parathyroid tumors (Guarnieri et al. 2017). Our results show that in parathyroid tumor cells, miR-372 may dampen the Wnt pathway through upregulation of the Wnt-inhibitor DKK1 and repression of cyclin D1 gene. This pattern of modulation is consistent with the observed cell cycle arrest in G1 phase of parathyroid tumor cultures.

Concluding, although the present study has some limitations such as the lack of normal parathyroid cells for functional experiments and the transient nature of the miR-372 overexpression, we show for the first time that the embryonic miR-372 is aberrantly expressed in scattered endocrine neoplastic cells of a consistent subset of aggressive parathyroid tumors, such as atypical adenomas and carcinomas. miR-372 aberrant expression could confer resistance to apoptosis in non-dividing parathyroid tumor cells where it is associated with increased PTH expression and in vivo PTH release.

Taken together, our data show that the aberrantly reactivated embryonic signaling governed by miR-372 plays a role in parathyroid tumorigenesis that may provide important novel insights into patients' stratification and treatment.

\section{Supplementary data}

This is linked to the online version of the paper at https://doi.org/10.1530/ ERC-17-0204.

\section{Declaration of interest}

The authors declare that there is no conflict of interest that could be perceived as prejudicing the impartiality of the research reported.

\section{Funding}

This study was supported by the Italian Minister of Health grant GR201102351626 (to V V), by the University of Milan Fondi 2015 Linea 2 (to S C) and by Ricerca Corrente IRCCS Policlinico San Donato and IRCCS Istituto Ortopedico Galeazzi (L4080) (to S C). M A is supported by a fellowship from the Doctorate School in Molecular and Translational Medicine from the University of Milan.

\section{References}

Arribas B, Cristobal E, Alcazar JA, Tardio J, Martinez-Montero JC, Polo JR, Carrion R, Gil L, Azanedo M, Rojas JM, et al. 2000 p53/ MDM2 pathway aberrations in parathyroid tumor: $\mathrm{p} 21$ (WAF-1) and
(2) 2018 Society for Endocrinology Published by Bioscientifica Ltd. Printed in Great Britain 
MDM2 are frequently overexpressed in parathyroid adenomas. Endocrine Pathology 11 251-257. (https://doi.org/10.1385/ EP:11:3:251)

Bondenson L, Grimelius L, De Lellis RA, Lloyd R, Akerstrom G, Larsson C, Arnold A, Eng C, Shane E \& Bilezikian JP. 2004 Parathyroid carcinoma. In Pathology and Genetics. Tumors of Endocrine Organs. WHO Classification of Tumors, pp 128-132. Eds RA DeLelli, RV Lloyd, PU Heltz \& C Eng. Lyon, France: IARC Press.

Buchwald PC, Akerström G \& Westin G 2004 Reduced p18INK4c, p21CIP1/WAF1 and p27KIP1 mRNA levels in tumours of primary and secondary hyperparathyroidism. Clinical Endocrinology $\mathbf{6 0}$ 389-393. (https://doi.org/10.1111/j.1365-2265.2004.01995.x)

Chen X, Hao B, Han G, Liu Y, Dai D, Li Y, Wu X, Zhou X, Yue Z, Wang L, et al. 2015 miR-372 regulates glioma cell proliferation and invasion by directly targeting PHLPP2. Journal of Cellular Biochemistry 116 225-232. (https://doi.org/10.1002/jcb.24949)

Cho WJ, Shin JM, Kim JS, Lee MR, Hong KS, Lee JH, Koo KH, Park JW \& Kim KS 2009 miR-372 regulates cell cycle and apoptosis of ags human gastric cell line through direct regulation of LATS2. Molecules and Cells 31 521-527. (https://doi.org/10.1007/s10059-009-0158-0)

Corbetta S, Vaira V, Guarnieri V, Scillitani A, Eller-Vainicher C, Ferrero S, Vicentini L, Chiodini I, Bisceglia M, Beck-Peccoz P, et al. 2010 Differential expression of microRNAs in human parathyroid carcinomas compared with normal parathyroid tissue. EndocrineRelated Cancer 17 135-146. (https://doi.org/10.1677/ERC-09-0134)

Di Leva G, Garofalo M \& Croce CM 2014 MicroRNAs in cancer. Annual Review of Pathology 9 287-314. (https://doi.org/10.1146/annurevpathol-012513-104715)

Eriksen AH, Andersen RF, Nielsen BS, Sørensen FB, Appelt AL, Jakobsen A \& Hansen TF 2016 Intratumoral heterogeneity of microRNA expression in rectal cancer. PLOS ONE 11 e0156919. (https://doi.org/10.1371/journal.pone.0156919)

Gartel AL \& Radhakrishnan SK 2005 Lost in transcription: p21 repression, mechanisms, and consequences. Cancer Research $\mathbf{6 5}$ 3980-3985. (https://doi.org/10.1158/0008-5472.CAN-04-3995)

Guan B, Welch JM, Sapp JC, Ling H, Li Y, Johnston JJ, Kebebew E, Biesecker LG, Simonds WF, Marx SJ, et al. 2016 GCM2-activating mutations in familial isolated hyperparathyroidism. American Journal of Human Genetics 99 1034-1044. (https://doi.org/10.1016/j. ajhg.2016.08.018)

Guan X, Zong ZH, Chen S, Sang XB, Wu DD, Wang LL, Liu Y \& Zhao Y 2017 The role of miR.372 in ovarian carcinoma cell proliferation. Gene 624 14-20. (https://doi.org/10.1016/i.gene.2017.04.043)

Guarnieri V, Muscarella LA, Verdelli C \& Corbetta S 2017 Alterations of DNA methylation in parathyroid tumors. Molecular and Cellular Endocrinology [epub]. (https://doi.org/10.1016/j.mce.2017.05.010)

Huang X, Huang M, Kong L \& Li Y 2015 miR-372 suppresses tumour proliferation and invasion by targeting IGF2BP1 in renal cell carcinoma. Cell Proliferation 48 593-599. (https://doi.org/10.1111/ cpr.12207)

Langroudi L, Jamshidi-Adegani F, Shafiee A, Rad SM, Keramati F, Azadmanesh K, Arefian E \& Soleimani M 2015 MiR-371-373 cluster acts as a tumor-suppressor-miR and promotes cell cycle arrest in unrestricted somatic stem cells. Tumour Biology 36 7765-7774. (https://doi.org/10.1007/s13277-015-3519-7)

Li G, Zhang Z, Tu Y, Jin T, Liang H, Cui G, He S \& Gao G 2013 Correlation of microRNA-372 upregulation with poor prognosis in human glioma. Diagnostic Pathology 81.

Li SS, Yu SL, Kao LP, Tsai ZY, Singh S, Chen BZ, Ho BC, Liu YH \& Yang PC 2009 Target identification of microRNAs expressed highly in human embryonic stem cells. Journal of Cellular Biochemistry 106 1020-1030. (https://doi.org/10.1002/jcb.22084)

Liu BL, Sun KX, Zong ZH, Chen S \& Zhao Y 2016 MicroRNA-372 inhibits endometrial carcinoma development by targeting the expression of the Ras homolog gene family member C (RhoC). Oncotarget 7 6649-6664. (https://doi.org/10.18632/oncotarget.13871)
McCoy KL, Seethala RR, Armstrong MJ, Nikiforova MN, Stang MT, Carty SE \& Yip L 2015 The clinical importance of parathyroid atypia: is long-term surveillance necessary? Surgery 158 929-936. (https:// doi.org/10.1016/j.surg.2015.06.022)

Medeiros LA, Dennis LM, Gill ME, Houbaviy, H, Markoulaki S, Fu D, White AC, Kirak O, Sharp PA, Page DC, et al. 2011 Mir-290-295 deficiency in mice results in partially penetrant embryonic lethality and germ cell defects. PNAS 108 14163-14168. (https://doi. org/10.1073/pnas.1111241108)

Qi J, Yu JY, Shcherbata HR, Mathieu J, Wang AJ, Seal S, Zhou W, Stadler BM, Bourgin D, Wang L, et al. 2009 microRNAs regulate human embryonic stem cell division. Cell Cycle 8 3729-3741. (https://doi.org/10.4161/cc.8.22.10033)

Rosenbluth EM, Shelton DN, Sparks AET, Devor E, Christenson L \& Van Voorhis BJ 2013 MicroRNA expression in the blastocyst. Fertility and Sterility 99 855-861. (https://doi.org/10.1016/j.fertnstert.2012.11.001)

Strauss WM, Chen C, Lee CT \& Ridzon D 2006 Nonrestrictive developmental regulation of microRNA gene expression. Mammalian Genome 17 833-840. (https://doi.org/10.1007/s00335-006-0025-7)

Stojadinovic A, Hoos A, Nissan A, Dudas ME, Cordon-Cardos C, Shaha AR, Brennan MF, Singh B \& Ghossein RA 2003 Parathyroid neoplasms: clinical, histopathological, and tissue microarray-based molecular analysis. Human Pathology 34 54-64. (https://doi. org/10.1053/hupa.2003.55)

Subramanyam D, Lamouille S, Judson RL, Liu JY, Bucay N, Derynck R \& Blelloch R 2011 Multiple targets of miR-302 and miR-372 promote reprogramming of human fibroblasts to induced pluripotent stem cells. Nature Biotechnology 29 443-448. (https://doi.org/10.1038/nbt.1862)

Suh MR, Lee Y, Kim JY, Kim SK, Moon SH, Lee JY, Cha KY, Chung HM, Yoon HS, Moon SY, et al. 2004 Human embryonic stem cells express a unique set of microRNAs. Developmental Biology 270 488-498. (https://doi.org/10.1016/j.ydbio.2004.02.019)

Tan JK, Tan E \& Gan SY 2014 Elucidating the roles of miR-372 in cell proliferation and apoptosis of nasopharyngeal carcinoma TW01 cells. Experimental Oncology 36 170-173.

Tian RQ, Wang XH, Hou LJ, Jia WH, Yang Q, Li YX, Liu M, Li X \& Tang H 2011 MicroRNA-372 is down-regulated and targets cyclindependent kinase 2 (CDK2) and cyclin A1 in human cervical cancer, which may contribute to tumorigenesis. Journal of Biological Chemistry 286 25556-25563. (https://doi.org/10.1074/jbc. M111.221564)

Tu HF, Chang KW, Cheng HW \& Liu CJ 2015 Upregulation of miR-372 and miR-373 associates with lymph node metastasis and poor prognosis of oral carcinomas. Laryngoscope 125 E365-E370. (https:// doi.org/10.1002/lary.25464)

Vaira V, Elli F, Forno I, Guarnieri V, Verdelli C, Ferrero S, Scillitani A, Vicentini L, Cetani F, Mantovani G, et al. 2012a The microRNA cluster C19MC is deregulated in parathyroid tumours. Journal of Molecular Endocrinology 49 115-124. (https://doi.org/10.1530/JME-11-0189)

Vaira V, Faversani A, Dohi T, Montorsi M, Augello C, Gatti S, Coggi G, Altieri DC \& Bosari S 2012b miR-296 regulation of a cell polarity-cell plasticity module controls tumor progression. Oncogene 31 27-38. (https://doi.org/10.1038/onc.2011.209)

Vaira V, Verdelli C, Forno I \& Corbetta S 2016 MicroRNAs in parathyroid physiopathology. Molecular and Cellular Endocrinology 456 9-15. (https://doi.org/10.1016/j.mce.2016.10.035)

Verdelli C, Avagliano L, Guarnieri V, Cetani F, Ferrero S, Vicentini L, Beretta E, Scillitani A, Creo P, Bulfamante GP, et al. 2017 Expression, function and regulation of the embryonic transcription factor TBX1 in parathyroid tumors. Laboratory Investigation 97 1488-1499. (https://doi.org/10.1038/labinvest.2017.88)

Voorhoeve PM, le Sage C, Schrier M, Gillis AJ, Stoop H, Nagel R, Liu YP, van Duijse J, Drost J, Griekspoor A, et al. 2006 A genetic screen implicates miRNA-372 and miR-373 as oncogenes in testicular germ cell tumors. Cell 124 1169-1181. (https://doi.org/10.1016/j. cell.2006.02.037)
(2) 2018 Society for Endocrinology Published by Bioscientifica Ltd. Printed in Great Britain 
Wang Y, Baskerville S, Shenoy A, Babiarz JE, Baehner L \& Blelloch R 2008 Embryonic stem cell-specific microRNAs regulate the G1-S transition and promote rapid proliferation. Nature Genetics $\mathbf{4 0}$ 1478-1483. (https://doi.org/10.1038/ng.250)

Wu G, Wang Y, Lu X, He H, Liu H, Meng X, Xia S, Zheng K \& Liu B 2015 Low miR-372 expression correlates with poor prognosis and tumor metastasis in hepatocellular carcinoma. BMC Cancer 15182. (https://doi.org/10.1186/s12885-015-1214-0)

Yamashita S, Yamamoto H, Mimori K, Nishida N, Takahashi H, Haraguchi N, Tanaka F, Shibata K, Sekimoto M, Ishii H, et al. 2012
MicroRNA-372 is associated with poor prognosis in colorectal cancer. Oncology 82 205-212. (https://doi.org/10.1159/000336809)

Zhao B, Ye X, Yu J, Li L, Li W, Li S, Yu J, Lin JD, Wang CY, Chinnaiyan AM, et al. 2008 TEAD mediates YAP-dependent gene induction and growth control. Genes and Development 22 1962-1971.

Zhou A-D, Diao L-T, Xu H, Xiao Z-D, Li J-H, Zhou H \& Qu L-H 2012 $\beta$-Catenin/LEF1 transactivates the microRNA-371-373 cluster that modulates the Wnt/ $\beta$-catenin-signaling pathway. Oncogene 31 2968-2978. (https://doi.org/10.1038/onc.2011.461)

Received in final form 26 April 2018

Accepted 1 May 2018

Accepted Preprint published online 3 May 2018
(C) 2018 Society for Endocrinology Published by Bioscientifica Ltd. Printed in Great Britain 